\title{
MIDPI
}

\section{Accelerating the DszD enzyme for the Biodesulfurization of Crude Oil and Derivatives}

\author{
Pedro Ferreira ${ }^{a}$, Sérgio Sousa ${ }^{b}$, Pedro A. Fernandes ${ }^{a}$, Maria João Ramos $^{a *}$ \\ a UCIBIO@REQUIMTE - Faculdade de Ciências da Universidade do Porto \\ ${ }^{b}$ UCIBIO@REQUIMTE - Faculdade de Medicina da Universidade do Porto
}

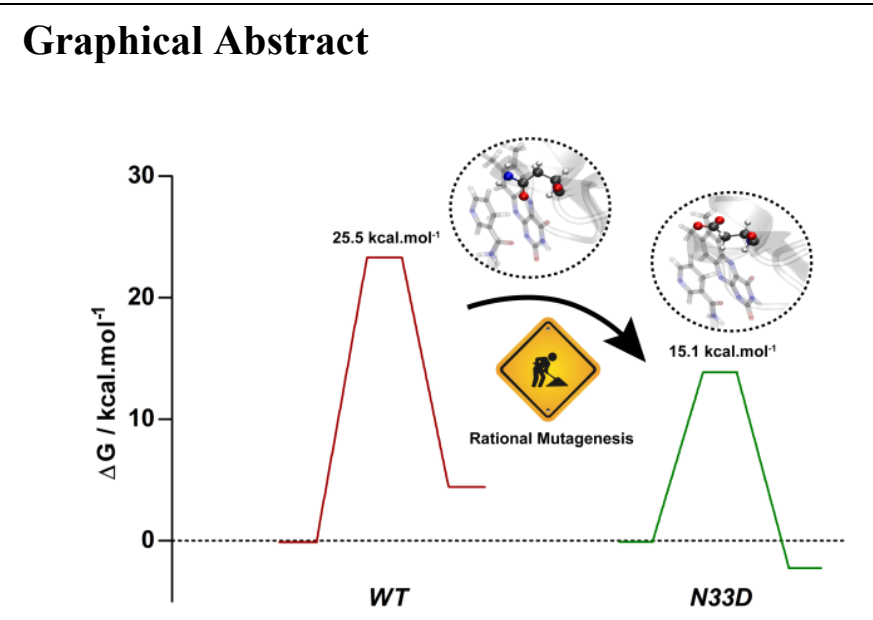

\begin{abstract}
.
It is known that fossil fuel combustion is one of the main environmental problems of the modern era, and the sulfur content of crude oil [1] contributes heavily to this. One of the main sulphurous compounds present in crude oil is dibenzothiophene (DBT). Due to its harmfulness, several governments around the world have been imposing stricter restrictions regarding the sulfur content in fossil fuels. The desulfurization of crude oil is currently carried out using costly chemical processes. One alternative to these costly chemical processes involves the use of specific microorganisms, such as Rhodococcus erythropolis, capable of utilizing DBT as a sole source of sulfur. The process carried out by $R$. erytrhopolis is called the $4 S$ pathway and is conducted by the action of four enzymes of the dibenzothiophene desulfurization enzymes ( $d s z$ ) family. DszA, DszB, DszC and DszD. The major limitation of this pathway is the slow catalytic rates of the four enzymes, which limits its application in industry. The enhancement of the catalytic power of enzymes is a subject of enormous interest both for science and for industry. The latter, in particular, due to the vast applications enzymes can have in industrial processes. In this work, we sought to enhance the turnover rate of DszD from Rhodococcus erythropolis, a NADH-FMN oxidoreductase
\end{abstract}


responsible to supply $\mathrm{FMNH}_{2}$ to $\mathrm{DszA}$ and $\mathrm{DszC}$ in the biodesulfurization process of crude oil, the $4 S$ pathway. For that purpose, we replaced the wild type spectator residue of the rate-limiting step of the reduction of FMN to $\mathrm{FMNH}_{2}$ catalysed by DszD, known to have an important role in its energetic profile, with all the natural occurring amino acids, one at a time, using computational methodologies, and repeated the abovementioned reaction with each mutant. To calculate the different free energy profiles, one for each mutated model, we applied quantum mechanical methods (namely DFT) within an ONIOM scheme. The free energy barriers obtained for the different mutated models varied

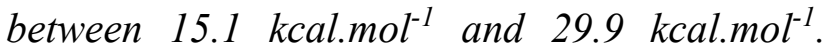
Multiple factors contributed to the different $\Delta G s$. The most relevant were electrostatic interactions and the induction of a favourable alignment between substrate and cofactor. These results confirm the great potential that chirurgic mutations have to increase the catalytic power of DszD in relation to the wt enzyme [2].

\section{References}

[1] N. Kamali, M. Tavallaie, B. Bambai, A. A. Karkhane and M. Miri, Biotechnol Lett, 2010, 32, 921927.

[2] P. Ferreira, S. F. Sousa, P. A. Fernandes, M. J. Ramos, Chem. Eur. J. 2017, 23, 17231. https://doi.org/10.1002/chem.201704057 